\title{
Discharging dynamics of topological batteries
}

\author{
Vishal P. Patil,,${ }^{1 *}$ Žiga Kos $\odot,{ }^{1,2, *}$ Miha Ravnik,,${ }^{2,3}$ and Jörn Dunkel ${ }^{1}$ \\ ${ }^{1}$ Department of Mathematics, Massachusetts Institute of Technology, 77 Massachusetts Avenue, Cambridge, Massachusetts 02139, USA \\ ${ }^{2}$ Faculty of Mathematics and Physics, University of Ljubljana, Jadranska 19, 1000 Ljubljana, Slovenia \\ ${ }^{3}$ Condensed Matter Physics Department, J. Stefan Institute, Jamova 39, 1000 Ljubljana, Slovenia
}

(Received 24 February 2020; accepted 14 October 2020; published 6 November 2020)

\begin{abstract}
Topological constraints have long been known to provide efficient mechanisms for localizing and storing energy across a range of length scales, from knots in DNA to turbulent plasmas. Despite recent theoretical and experimental progress on the preparation of topological states, the role of topology in the discharging dynamics is not well understood. Here we investigate robust topological energy release protocols in two archetypal soft systems through simulations of 238 knotted elastic fibers and three-dimensional liquid crystals across a range of different topologies. By breaking the elastic fiber or switching the liquid crystal surface anchoring, such topological batteries can perform mechanical work or drive fluid flows. Our study reveals topologically resonant states for which energy release becomes superslow or superfast. Owing to their intrinsic stability we expect such tunable topological batteries to have broad applications to storage and directed release of energy in soft matter.
\end{abstract}

DOI: 10.1103/PhysRevResearch.2.043196

\section{INTRODUCTION}

Topological protection provides a robust means for storing and controlling energy, an effect widely used in a variety of biological and physical systems [1-6]. On small scales, knotted topologies play important functional roles [7] in the behavior of tangled DNA, proteins, and polymers [8-12]. In continuum systems, foundational work on topology has revealed the origin of helicity conservation in classical $[2,13]$ and complex fluids [14] and the dynamo effect in turbulent plasmas $[15,16]$. Structured continua such as liquid crystal fluids are a rich source of emergent topological phenomena, from interacting defects $[17,18]$ to knotted field configurations $[3,19,20]$. Recent experimental advances in mechanical lattices [21,22] and soft robotics [23] bring the question of topologically tunable designer materials into the experimentally accessible realm. Although the study of topological modes [24] has dramatically improved our understanding of soft matter, harnessing topology to perform useful work such as driving flows $[17,25,26]$ continues to present fundamental challenges.

Here we study how topology affects energy release dynamics in knotted elastic fibers [27-29] and nematic liquid crystals [19], demonstrating two distinct realizations of a topological battery. In both cases, topology-mediated buckling and instability phenomena underlie the discharging rates and functional capabilities of the batteries. Knotted filaments

\footnotetext{
*These authors contributed equally to this work.

Published by the American Physical Society under the terms of the Creative Commons Attribution 4.0 International license. Further distribution of this work must maintain attribution to the author(s) and the published article's title, journal citation, and DOI.
}

present an intuitive mechanical realization of a topological battery: By initializing closed elastic loops in tight knotted states $[28,30,31]$ with varying twist $[32,33]$, energy may be stored robustly (see Fig. 1 below). Cutting the knot at the point of maximum stress results in a controlled topology-dependent energy discharge. Transferring this idea to liquid crystals, nematic batteries can be realized by imprinting topologically nontrivial field configurations, which are energetically stabilized through the anchoring of the nematic orientational director field to a colloidal surface [3,19,20]. Energy is then released by optically changing the anchoring profile. Using three-dimensional (3D) numerical simulations, we explore the energy discharge dynamics in elastic fibers and liquid crystals across a range of different knot types and topological charges. For both systems, we find special topologically resonant states that are characterized by superslow or superfast energy release, exemplifying control of discharging dynamics through topology.

\section{ENERGY RELEASE DYNAMICS IN ELASTIC KNOTS}

\section{A. Kirchhoff model with contact friction}

Tying a knot in an elastic fiber and fusing together the free ends produces our first example of a topological battery. By twisting the free ends before fusing, knotted batteries can be studied along the two axes of knot type and twist density (Fig. 1). We describe this system using the Kirchhoff model for elastic rods together with contact friction [29,34]. The fibers have natural length $L$, radius $h$, and circular cross section with moment of inertia $I=\pi h^{4} / 4$ and crosssectional area $A=\pi h^{2}$. The state of the fiber is defined by its centerline curve $\boldsymbol{x}(s)$ and an orthonormal material frame, $\left\{\mathbf{d}_{1}(s), \mathbf{d}_{2}(s), \mathbf{d}_{3}(s)\right\}$, constrained by $\boldsymbol{x}^{\prime} \times \mathbf{d}_{3}=\mathbf{0}$, where $s$ is the arc length parameter of the unstretched fiber. The elastic 


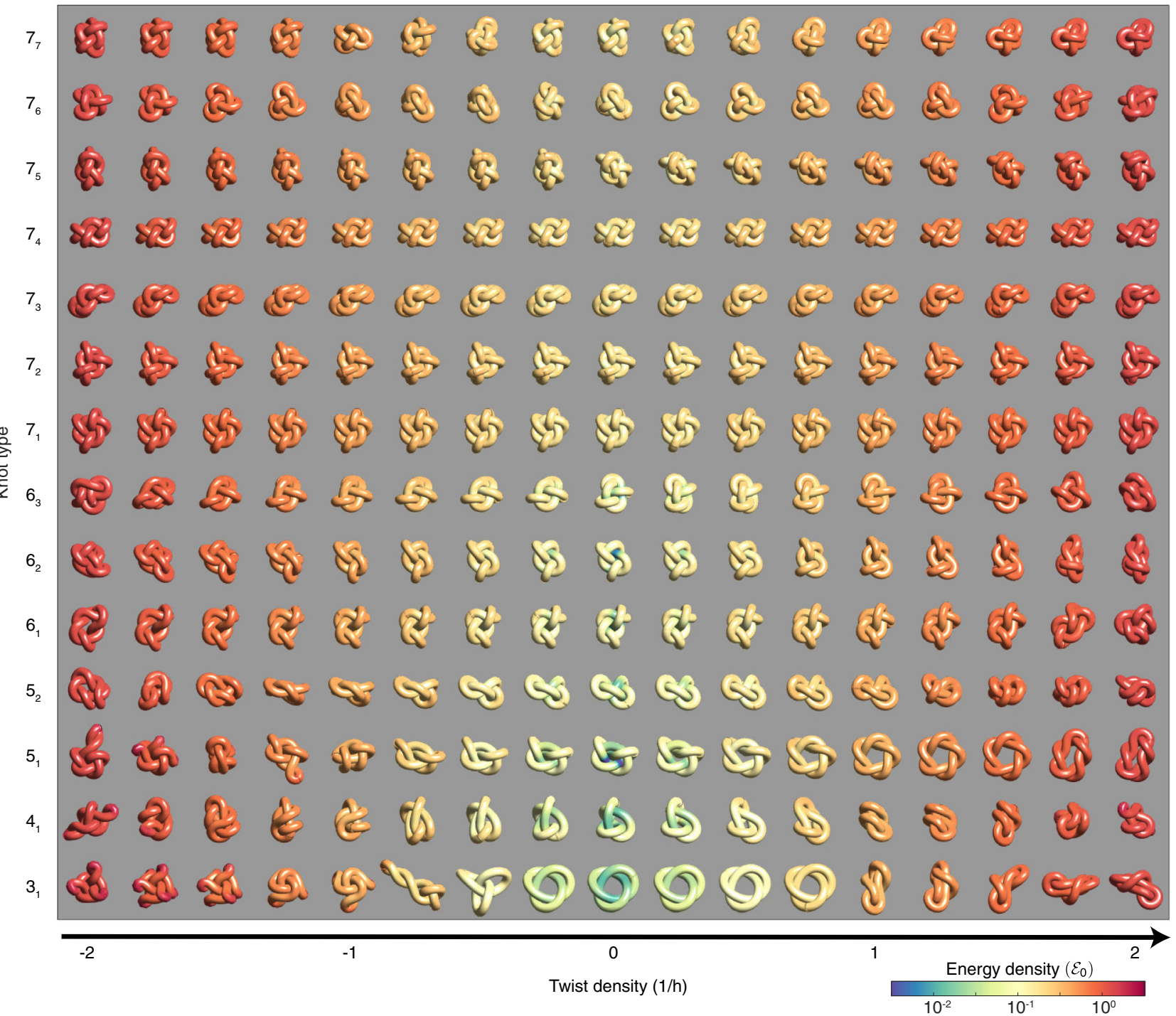

FIG. 1. Knotted topological batteries were simulated for 238 initial configurations, representing 17 different twist densities for 14 knot types. Simulation parameters: $\alpha^{-1}=1.3, \gamma=5, L / h=50$.

energy depends on the geometric curvature $\kappa$, twist density $\theta^{\prime}=\mathbf{d}_{\mathbf{1}}{ }^{\prime} \cdot \mathbf{d}_{\mathbf{2}}$, and stretch $\left|\boldsymbol{x}^{\prime}\right|$ :

$$
\mathcal{E}=\frac{E_{b} A}{2} \int_{0}^{L} d s\left[\frac{1}{4} h^{2}\left(\kappa^{2}+\alpha \theta^{\prime 2}\right)+\gamma\left(\left|\boldsymbol{x}^{\prime}\right|-1\right)^{2}\right],
$$

where $E_{b}$ is the bending modulus, $E=\gamma E_{b}$ is the Young's modulus, and $v=\alpha^{-1}-1$ is the Poission's ratio. To describe the dynamics, we define $\boldsymbol{\Omega}=\left(\dot{\mathbf{d}}_{1} \cdot \mathbf{d}_{2}\right) \mathbf{d}_{3}$. The governing equations follow from extremizing (1) and assuming viscous damping forces and friction dominate inertial terms:

$$
\begin{aligned}
\mathbf{F}^{\prime} & =-\eta A \dot{\boldsymbol{x}}^{\prime \prime}-\boldsymbol{f}_{\text {fric }}, \\
\mathbf{M}^{\prime}+\boldsymbol{x}^{\prime} \times \mathbf{F} & =-2 \eta I\left(\boldsymbol{\Omega}^{\prime \prime} \cdot \mathbf{d}_{\mathbf{3}}\right) \mathbf{d}_{\mathbf{3}} .
\end{aligned}
$$

$\mathbf{F}$ and $\mathbf{M}$ are the internal force and moment in the rod, respectively, $\boldsymbol{f}_{\text {fric }}$ is a friction force density, and $\eta$ is a damping parameter. Constitutive laws yield expressions for $\mathbf{F}$ and $\mathbf{M}$ :

$$
\begin{aligned}
\mathbf{M} & =E_{b} I\left(\mathbf{d}_{\mathbf{3}} \times \mathbf{d}_{\mathbf{3}}{ }^{\prime}+\alpha \theta^{\prime} \mathbf{d}_{3}\right), \\
\mathbf{F} \cdot \mathbf{d}_{\mathbf{3}} & =E A\left(\left|\boldsymbol{x}^{\prime}\right|-1\right) .
\end{aligned}
$$

The friction force density is based on a friction model that has been experimentally validated [29]:

$$
\boldsymbol{f}_{\text {fric }}=\eta_{f} A \int d s^{\prime} \Theta\left(2 h-\left|\boldsymbol{x}(s)-\boldsymbol{x}\left(s^{\prime}\right)\right|\right) \frac{\dot{\boldsymbol{x}}(s)-\dot{\boldsymbol{x}}\left(s^{\prime}\right)}{\left|\boldsymbol{x}(s)-\boldsymbol{x}\left(s^{\prime}\right)\right|^{3}} .
$$

In contrast to Ref. [29], we take $\eta_{f} / \eta=0.1$ (instead of setting $\left.\eta_{f}=\eta\right)$ and neglect the effect of torsional friction. The intrinsic energy density, $\mathcal{E}_{0}=E_{b} h^{2}$, and energy, $E_{0}=E_{b} h^{2} L$, of the system correspond to the bending energy associated with $\kappa \sim 1 / h$. We choose an intrinsic timescale, $T_{0}=2 L \eta / h E_{b}$, as an intermediate scale between the relaxation times of pure twist, $T_{t w}=\eta / \alpha E_{b}$, and pure bending, $T_{b}=2 L^{2} \eta / \pi^{2} h^{2} E_{b}$. The model applies to a range of different materials; a specific candidate system is thin lubricated silicone fibers with $\alpha^{-1} \sim 1.3, \gamma \sim 5, \eta \sim 10 \mathrm{kPas}, E_{b} \sim 10 \mathrm{MPa}$.

\section{B. Charging}

Charging knotted filaments with twist results in a series of topology-mediated buckling instabilities [Fig. 2(a)]. The twist 

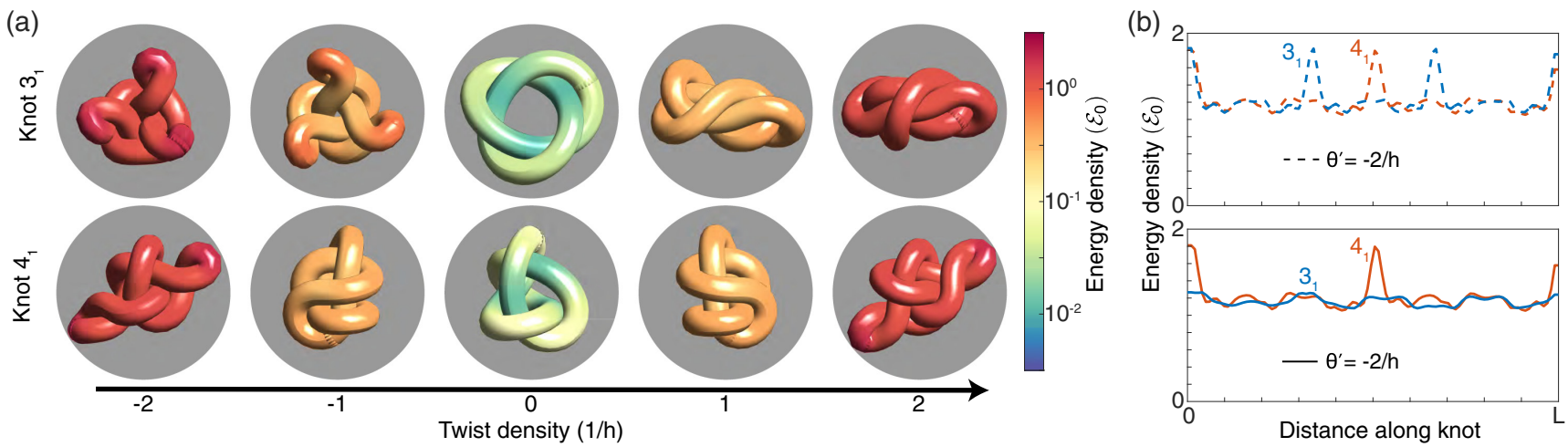

FIG. 2. Charging topological batteries with knot tying and twist, illustrated for two knot types. (a) Energy storage in elastic batteries depends on both knot type and initial twist density. The chiral trefoil knot $\left(3_{1}\right.$, top) exhibits different buckled states at positive and negative twist, whereas the achiral figure-of-eight knot $\left(4_{1}\right.$, bottom) does not. (b) Chirality-dependent buckling leads to different energy density profiles for the trefoil knot when charged with positive twist $\left(\theta^{\prime}=2 / h\right)$ and negative twist $\left(\theta^{\prime}=-2 / h\right)$. Simulation parameters: $\alpha^{-1}=1.3, \gamma=$ $5, L / h=50$.

in the buckled state is typically lower than the initial twist supplied to the prebuckled state [35]. The evolution of the total twist in the fiber, $T w$, follows from relating spatial and time derivatives of the frame:

$$
\frac{d}{d t} T w=\left[\mathbf{\Omega} \cdot \mathbf{d}_{3}\right]_{0}^{L}+\int_{0}^{L} d s \mathbf{d}_{3} \cdot\left(\mathbf{d}_{3}^{\prime} \times \dot{\mathbf{d}}_{3}\right) .
$$

When the fiber is closed, the first term on the right-hand side vanishes. Although initializing a fiber with a specific twist requires a time-dependent torque application protocol, the amount of buckling provides an indicator of the twist charge within the battery, for a given knot type. For example, twist produces initial battery states with varying postbuckled geometries [Fig. 2(a)] and energy densities [Fig. 2(b)]. Additionally, twist is sensitive to certain underlying topological properties. In our simulations, chiral knots generally exhibit handedness-dependent buckling [Fig. 2(a), top], whereas achiral knots tend to buckle independently of twist handedness [Fig. 2(a), bottom].

\section{Discharging dynamics and topological resonance}

As knotted mechanical batteries unravel, the competition between bending and twisting energies reveals a landscape of topological resonances, where certain initial states lead to super slow energy discharge. After being broken at the point of maximum stress, different transient, metastable states occur [see Fig. 3(a) and the movie in the Supplemental Material [36]]. The discharge dynamics separate into a bendingdominated regime and a twist-dominated regime, as evident from the initial state of the battery [Fig. 3(b)]. The crossover between the two regimes corresponds to the scaling of strains associated with twisting and bending. From (1), the characteristic strains for tightly knotted configurations are $\varepsilon_{b} \sim h \kappa \sim 1$ for bending and $\varepsilon_{t w} \sim h \theta^{\prime} \sim h T w / L$ for twisting. As a result, twist dominates the discharge dynamics at high twist densities with $\theta^{\prime} h>1$ [Figs. 3(c)-3(e)]. Since the effects of varying knot type are more naturally related to the bending energy, twist can be thought of as washing out topology. Indeed, at high twist, $\theta^{\prime} h>1$, the batteries discharge quickly, indepen- dent of knot type [Figs. 3(d) and 3(e)]. By contrast, at low twist, knot topology essentially determines the discharging dynamics [Fig. 3(e)]. In particular, select knots exhibit extremely long discharge times [Figs. 3(d) and 3(e)]. We can explain these slow topological resonances by considering the mechanisms by which knots release twist and bending energy. Bending forces point in the $\mathbf{d}_{3}^{\prime}$ direction, which lies in the fiber's local osculating plane, spanned by $\mathbf{d}_{3}$ and $\mathbf{d}_{3}^{\prime}$. From (3), twist changes when $\dot{\mathbf{d}}_{3}$ has a component in the $\mathbf{d}_{\mathbf{3}} \times \mathbf{d}_{\mathbf{3}}{ }^{\prime}$ direction; twist relaxation therefore pushes the fiber out of plane. The topologically resonant slow knots can thus be thought of as maximally nonplanar and therefore self-confining.

\section{ENERGY RELEASE DYNAMICS IN NEMATIC FLUIDS}

\section{A. Nematodynamic model}

Nematic fluids containing spherical colloidal particles [19] enable another construction of a topological battery. The effective topological charge of the particle is determined by its prescribed liquid crystal anchoring profile, which may be optically changed $[37,38]$ to release energy. We model the nematic system with a tensor order parameter $\mathbf{Q}$. The largest eigenvalue and its eigenvector determine the degree of order $S$ and the director $\mathbf{n}$. The free energy, $F=\int f d V$, is given by

$$
f=\frac{A}{2} Q_{i j} Q_{j i}+\frac{B}{3} Q_{i j} Q_{j k} Q_{k i}+\frac{C}{4}\left(Q_{i j} Q_{j i}\right)^{2}+\frac{L}{2}\left(\partial_{k} Q_{i j}\right)^{2} .
$$

Derivatives of $Q_{i j}$ in the free energy density describe the effective elastic behavior of the director field, where $L$ is the elastic constant, and $A, B$, and $C$ are parameters that tune the nematic phase behavior. Our 3D nematodynamic simulations [39] are based on the Beris-Edwards formulation of the Qtensor dynamics [40]:

$$
\begin{aligned}
\left(\partial_{t}+\right. & \left.u_{k} \partial_{k}\right) Q_{i j}+\Omega_{i k} Q_{k j}-Q_{i k} \Omega_{k j}=\Gamma H_{i j}+\frac{2 \chi}{3} D_{i j} \\
& +\chi\left(D_{i k} Q_{k j}+Q_{i k} D_{k j}\right)-2 \chi\left(Q_{i j}+\frac{\delta_{i j}}{3}\right) Q_{k l} W_{k l}
\end{aligned}
$$




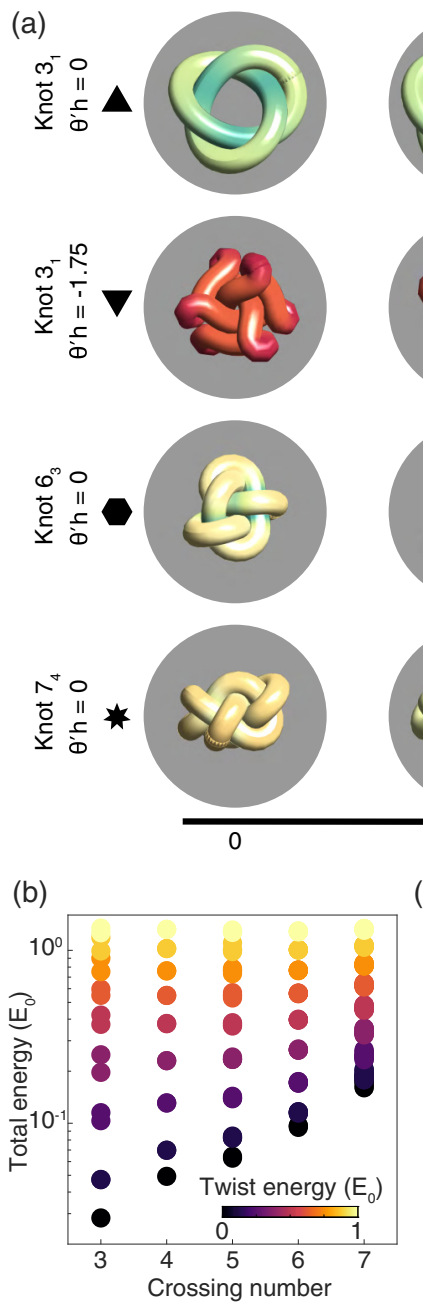

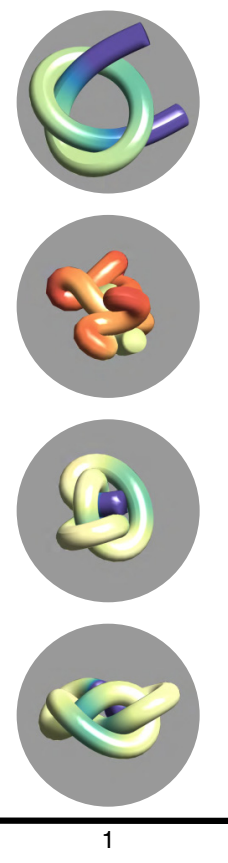

(c)
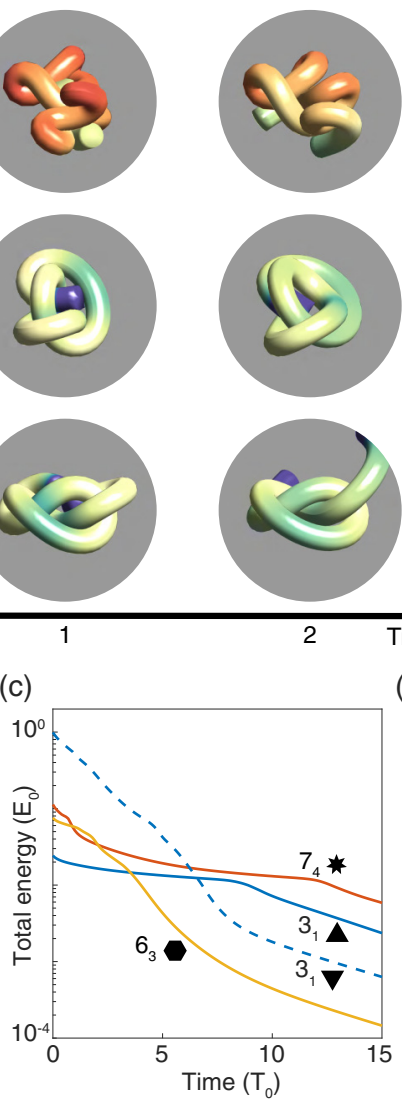
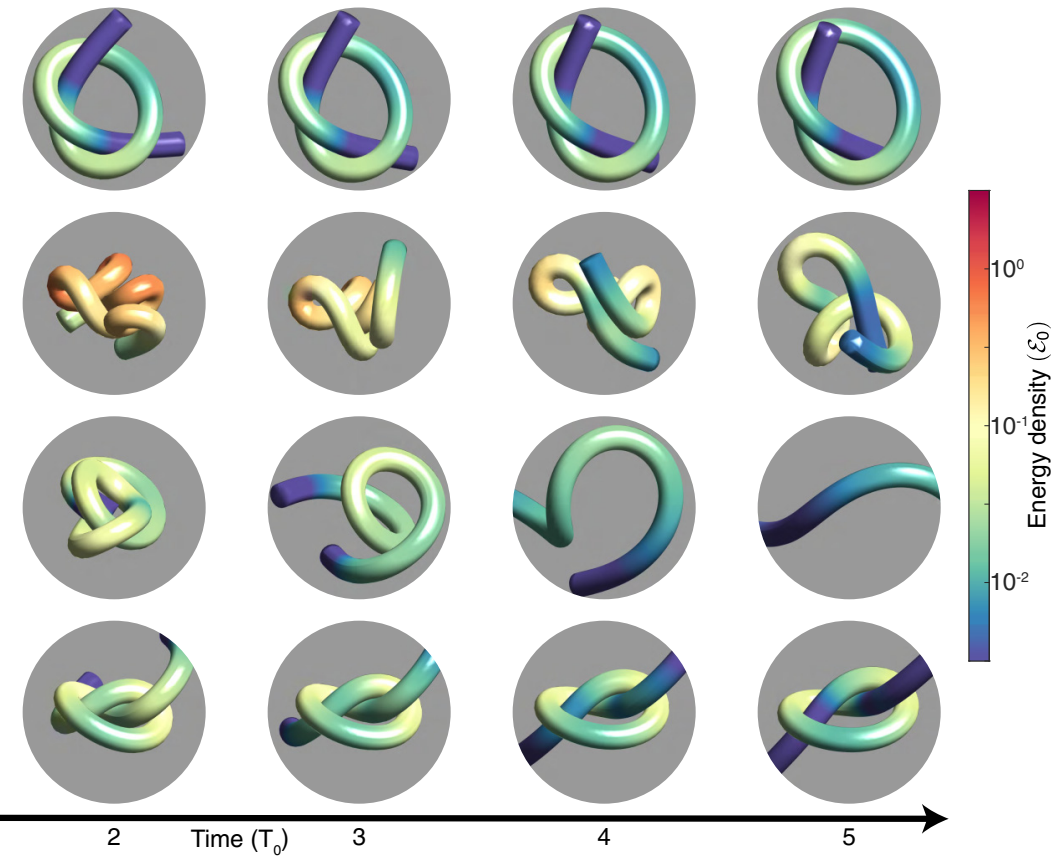

(d)

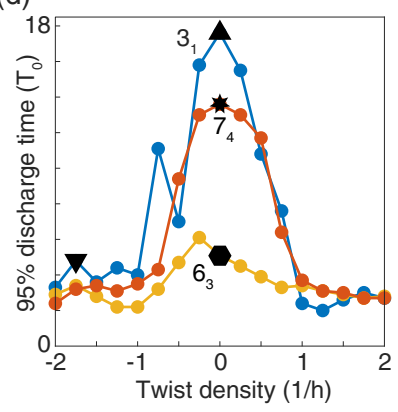

(e)

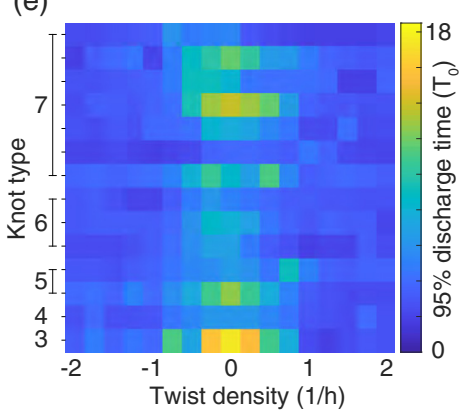

FIG. 3. Discharging knotted elastic fibers. (a) The relaxation dynamics depend strongly on knot type and twist state (see the simulation movie in the Supplemental Material [36]). (b) At low twist, the initial knot energy varies with knot type, while high twist negates the effect of topological changes. (c) Energy is released in phases as intermediate metastable topological states form and untie. Topology-dependent obstructions cause certain knot types to untie more slowly at zero twist $\left(3_{1}, 7_{4}\right)$. Higher twist states typically discharge faster, but the final discharge rates are set by the relaxed length of the fiber. (d) Topological resonances occur predominantly at low twist. (e) High twist leads to fast untying for all knot types. At scales where bending dominates, topology-dependent resonance effects become visible. Total number of initial knot configurations simulated for (e) is 238 (Fig. 1) using the algorithm from Refs. [29,30].

where $\mathbf{u}$ is fluid velocity, $\chi$ is the alignment parameter, and $\Gamma$ is the rotational viscosity coefficient. The molecular field $H_{i j}$ drives the system towards equilibrium, $H_{i j}=-\left(\delta F / \delta Q_{i j}\right)_{\mathrm{tr}}$, where $(\cdot)_{\text {tr }}$ denotes the traceless part. $D_{i j}$ and $\Omega_{i j}$ are the symmetric and antisymmetric parts of the velocity gradient tensor $W_{i j}=\partial_{i} u_{j}$. We model the nematic as an incompressible fluid with stress tensor

$$
\begin{aligned}
\sigma_{i j}= & 2 \chi\left(Q_{i j}+\frac{\delta_{i j}}{3}\right) Q_{k l} H_{k l}-\chi H_{i k}\left(Q_{k j}+\frac{\delta_{k j}}{3}\right) \\
& -\chi\left(Q_{i k}+\frac{\delta_{i k}}{3}\right) H_{k j}-\partial_{i} Q_{k l} \frac{\delta F}{\delta \partial_{j} Q_{k l}} \\
& +Q_{i k} H_{k j}-H_{i k} Q_{k j}+2 \eta D_{i j}-p \delta_{i j},
\end{aligned}
$$

where $p$ is the fluid pressure and $\eta$ is the isotropic viscosity. Defining the intrinsic length scale $\xi_{\mathrm{N}}=$ $\sqrt{L /\left(A+B S_{\text {eq }}+\frac{9}{2} C S_{\text {eq }}^{2}\right)}$ and timescale $\tau_{\mathrm{N}}=\xi_{\mathrm{N}}^{2} / \Gamma L$, the particle radius is set to $R=52.5 \xi_{\mathrm{N}}$ and the director field has relaxation timescale $\tau_{\mathrm{d}}=R^{2} / \Gamma L=2760 \tau_{\mathrm{N}}$. The phase parameters are set to $A=-0.19 L / \xi_{\mathrm{N}}^{2}, B=-2.34 L / \xi_{\mathrm{N}}^{2}$, and $C=1.91 L / \xi_{\mathrm{N}}^{2}$, The nematic is in the alignment regime $\chi=1$, with isotropic viscosity $\eta=1.38 \xi_{\mathrm{N}}^{2} / L \tau_{\mathrm{N}}$. Our $3 \mathrm{D}$ simulations were performed on a $200 \times 200 \times 150$ mesh with grid resolution $\Delta x=1.5 \xi_{\mathrm{N}}$ and time resolution $\Delta t=0.057 \tau_{\mathrm{N}}$. This Q-tensor-based formulation of nematohydrodynamics has been particularly effective for predicting and explaining experimental data measured in studies of entangled defect lines in nematic colloidal systems $[18,19]$, nematic colloids with variation of the particle or interface shape [41,42], patterned nematic interfaces [43], and microfluidics using nematic liquid crystals [17]. Such mesoscopic approaches can be directly expanded with other contributions and fluid mechanisms, including multiple elastic constants, driving of surface anchoring, and the effects of other possible mechanical or external fields. In addition, 
(a)

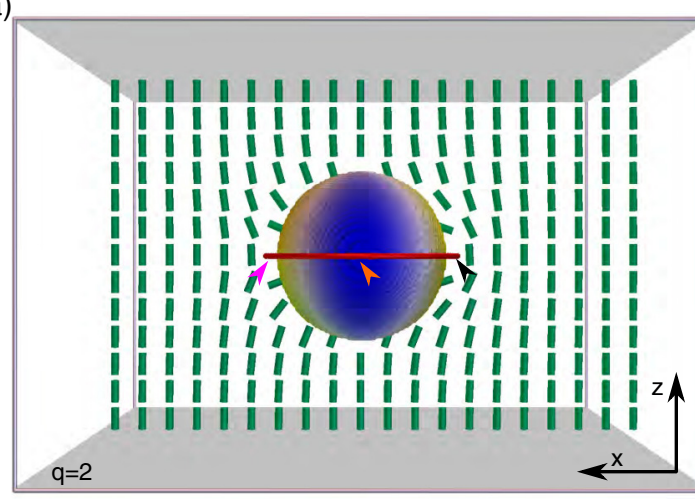

(b)

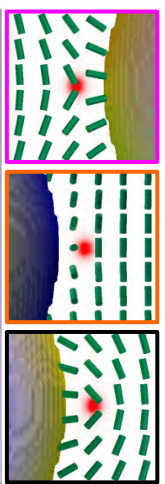

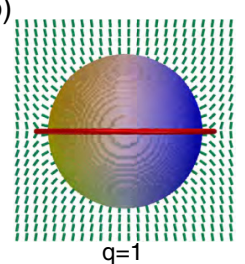

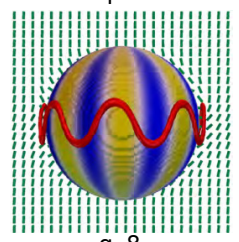

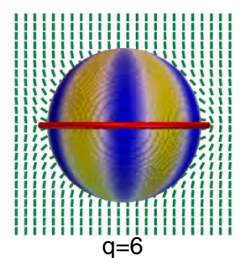

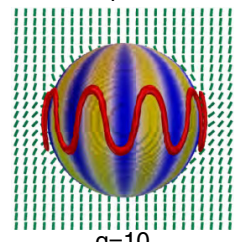

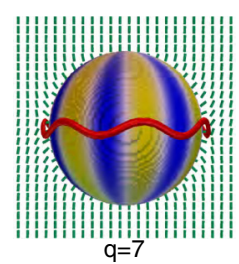

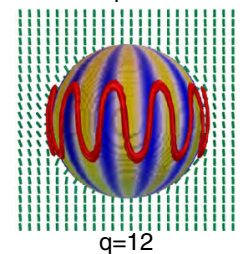

(e) $\times 10^{2}$
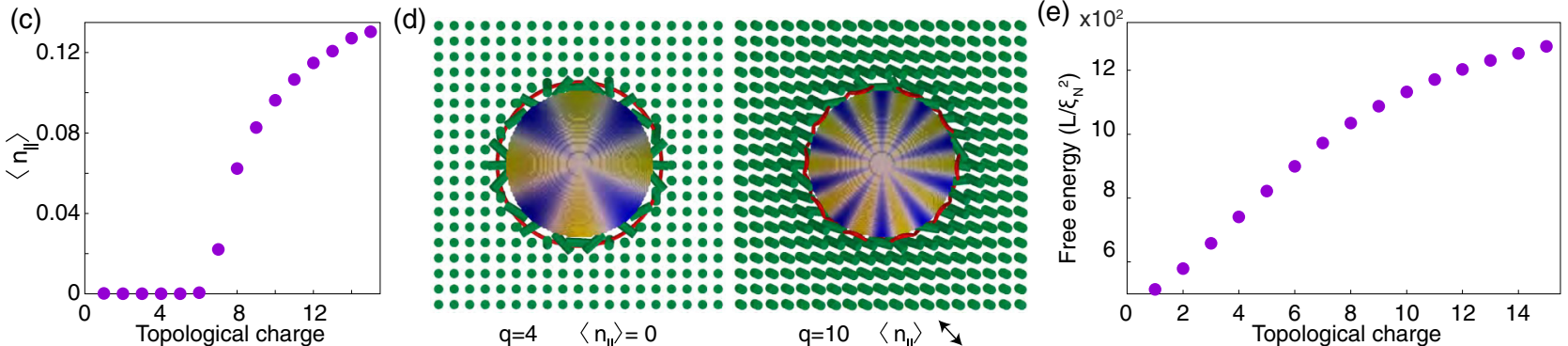

FIG. 4. Charging topological batteries in nematic fluids. (a) The battery consists of a spherical colloidal particle confined between two plates (gray), with topologically nontrivial anchoring profile, $\mathbf{n}_{q}(\theta, \phi)$, resulting in an effective topological charge, $q$, for the particle. The particle is colored from blue to yellow as the horizontal component of $\mathbf{n}_{q}$ varies from -1 to 1 . The director field (green rods) contains a defect ring (red isosurface at $S=0.35$ ) of varying cross-sectional structure (color-coded insets). (b) The defect ring buckles for $q \geqslant 7$ into a loop with $q$ maxima and minima. (c, d) Above the instability threshold $(q \geqslant 7)$, a nonzero mean director component emerges in the horizontal plane ( $x y)$ of the sample. (e) The total free energy of the nematic colloid is dependent on the topological charge of the nematic structure. See Sec. III A for simulation parameters.

surface-contributed timescales could also be controlled through in-plane switching methods [44].

\section{B. Charging}

Nematic topological batteries are charged by imposing a topologically nontrivial director field anchoring profile on the surface of a spherical particle given by $\mathbf{n}_{q}(\theta, \phi)=$ ( $\sin \theta \cos q \phi, \sin \theta \sin q \phi, \cos \theta$ ). Here $\theta$ and $\phi$ are the polar and azimuthal angles, and the integer $q$ sets the effective topological charge of the particle [45]. The system is placed in a cell with strong perpendicular anchoring of the nematic director on the top and bottom boundary [Fig. 4(a)]. The bulk nematic structure counteracts the topological charge of the particle by forming a defect ring [46], known as a Saturn ring for $q=1$. The director field in the cross sections of the defect ring varies continuously between $+1 / 2,-1 / 2$, and twisted profiles [Fig. 4(a)]. For small $q$, the defect ring lies on the equatorial plane of the particle [Figs. 4(a) and 4(b)]. As $q$ increases, the gradients of the director field increase, eventually causing the defect loop to buckle at a critical $q_{c}$ depending on colloid size. The resulting oscillatory defect profiles have wavelength matching that of the anchoring profile and amplitude increasing with $q$ [Fig. 4(b)]. The buckling transition is characterized by the average horizontal director field, $\left\langle\mathbf{n}_{\|}\right\rangle$, which is zero for $q<q_{c}$ and positive for $q \geqslant q_{c}$ [Figs. 4(c) and 4(d)]. The total free energy, $F$, however, strictly increases with $q$ [Fig. 4(e)]. Topology thus determines the distribution of energy throughout different modes, along with the total energy.

\section{Discharging dynamics and topological resonance}

Energy partitioning within the charged nematic batteries causes topological resonances in the discharge dynamics. The relaxation process is triggered by switching off the anchoring on the spherical particle, allowing the director field to take any orientation at its surface [Fig. 5(a)]. The initial reorientation of the director field is accompanied by the shrinking of the defect ring until it is annihilated on the particle surface. The continued reorientation of the director field towards the equilibrium homogeneous vertical structure drives a complex flow pattern [Fig. 5(a)]. During the typical director relaxation time, $\tau_{\mathrm{d}}$, the energy decreases by several orders of magnitude [Fig. 5(b)]. While the initial energy release rate increases with initial battery energy and topological charge $q$ [Fig. 5(c)], the asymptotic release rates show characteristic resonance peaks [Fig. 5(d)]. In particular, we observe superfast discharge rates for $q$ close to $q_{c}$ [Fig. 5(d)]. The return to slow relaxation for $q \gg q_{c}$ is caused by the formation of the long-scale deformation mode with $\left\langle\mathbf{n}_{\|}\right\rangle>0$ [Figs. 4(c) and 4(d)], which obstructs fast discharge.

\section{CONCLUSIONS}

In both studied systems, topology and mechanics interact to produce long-lived states that are central to the observed topological resonances. For elastic batteries, these states are 

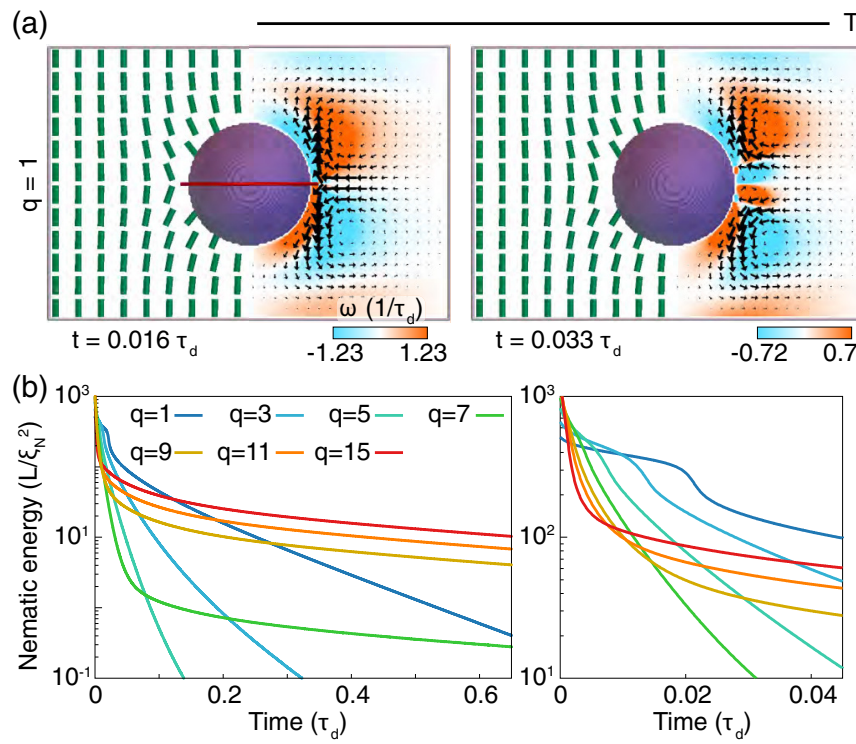

Time
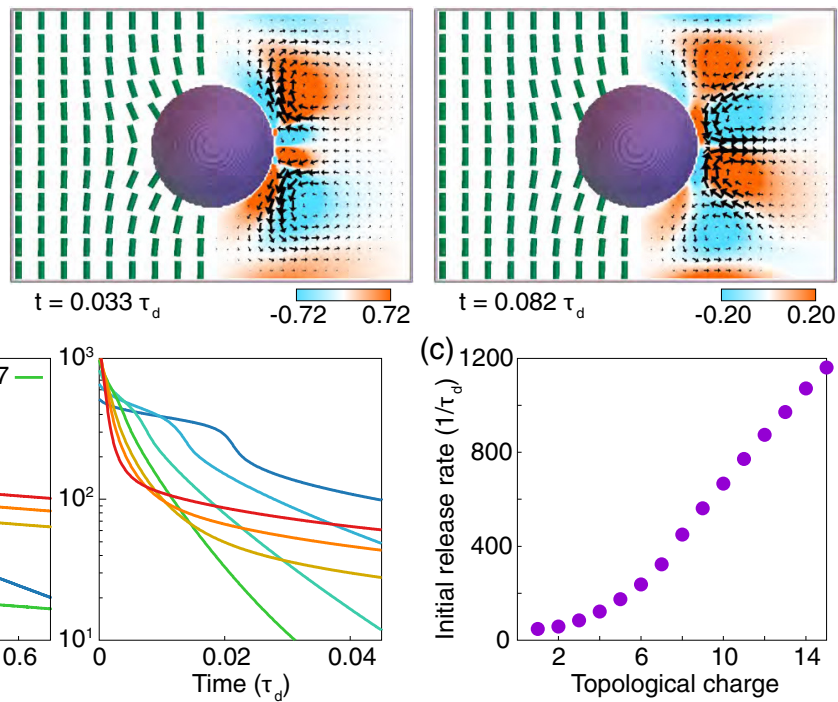

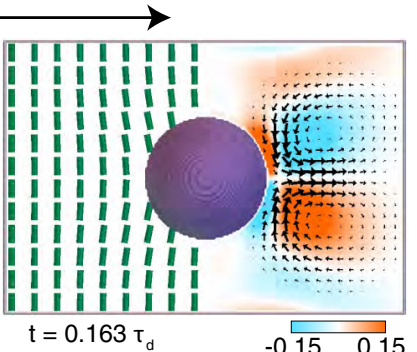

(d)

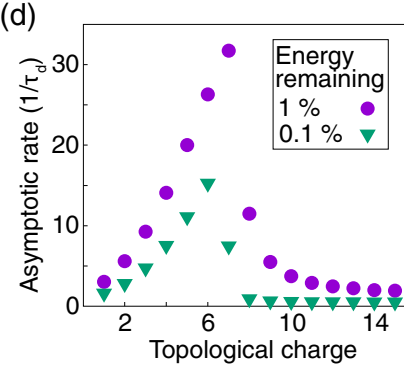

FIG. 5. Discharging nematic topological batteries. (a) As the orientational field (green rods) relaxes by changing its surface anchoring, the defect ring (red loop) is annihilated and drives a flow (black arrows) which changes direction over time. The flow vorticity $\omega$ measures the direction and magnitude of the flow. (b) Higher $q$ states have both larger initial energy and faster initial discharge. (c, d) The initial energy release rate, defined as the reciprocal of the $50 \%$ energy discharge time, is monotonic with topological charge. In contrast, the asymptotic release rates (d), given by the reciprocals of the $99 \%$ or $99.9 \%$ discharge times, display topological resonance. See Sec. III A for simulation parameters.

the intermediate knots that can form as the fiber unties. At small or zero twist, these configurations trap bending energy, leading to long relaxation times. Similarly, at sufficiently high topological charge, nematic batteries can store energy in the slow $\left\langle\mathbf{n}_{\|}\right\rangle$mode. Over long timescales, these modes make the pivotal contribution to the overall discharge rate. More generally, this principle of topologically activated slow modes can provide a conceptual framework for understanding topological resonances in other soft matter settings.

To conclude, knotted elastic fibers and topologically charged nematic fluids provide prime demonstrations of topological batteries. Both systems permit controlled triggering of energy release via fracture and photoalignment techniques. Our above analysis shows how elastic and nematic batteries can be topologically optimized to achieve slow or fast discharge. Owing to the inherent robustness of topological structures, the above ideas translate to a wide range of scales. The energy stored in topological batteries may be harnessed to drive flows or perform mechanical or electrical work, thus opening an avenue for topological control of soft systems.

\section{ACKNOWLEDGMENTS}

This work was supported by a MathWorks fellowship (V.P.), the Slovenian Research Agency (ARRS) through Grants No. L1-8135 (M.R.), No. P1-0099 (M.R. and Ž.K.), and No. N1-0124 (Ž.K.), the James S. McDonnell Foundation (J.D.), and the Robert E. Collins Distinguished Scholar Fund (J.D.).
[1] P. Virnau, L. A. Mirny, and M. Kardar, Intricate knots in proteins: Function and evolution, PLOS Comput. Biol 2, e122 (2006).

[2] H. K. Moffatt, The degree of knottedness of tangled vortex lines, J. Fluid Mech 35, 117 (1969).

[3] B. Senyuk, Q. Liu, S. He, R. D. Kamien, R. B. Kusner, T. C. Lubensky, and I. I. Smalyukh, Topological colloids, Nature (London) 493, 200 (2013).

[4] R. E. Goldstein, H. K. Moffatt, and A. I. Pesci, Topological constraints and their breakdown in dynamical evolution, Nonlinearity 25, R85 (2012).

[5] D. Ferraro, M. Campisi, G. M. Andolina, V. Pellegrini, and M. Polini, High-Power Collective Charging of a SolidState Quantum Battery, Phys. Rev. Lett 120, 117702 (2018).
[6] J. Ren, P. Hänggi, B. Li et al., Berry-Phase-Induced Heat Pumping and its Impact on the Fluctuation Theorem, Phys. Rev. Lett. 104, 170601 (2010).

[7] L. H. Kauffman, Knots and Physics (World Scientific, Singapore, 2001), Vol. 1

[8] D. Marenduzzo, E. Orlandini, A. Stasiak et al., DNA-DNA interactions in bacteriophage capsids are responsible for the observed DNA knotting, Proc. Natl. Acad. Sci. USA 106, 22269 (2009).

[9] R. C. Lua and A. Y. Grosberg, Statistics of knots, geometry of conformations, and evolution of proteins, PLoS Comput. Biol 2, e45 (2006).

[10] E. J. Rawdon, K. C. Millett, and A. Stasiak, Subknots in ideal knots, random knots, and knotted proteins, Sci. Rep. 5, 8928 (2015). 
[11] A. Stasiak, V. Katritch, J. Bednar, D. Michoud, and J. Dubochet, Electrophoretic mobility of dna knots, Nature (London) 384, 122 (1996).

[12] O. Gonzalez and J. H. Maddocks, Global curvature, thickness, and the ideal shapes of knots, Proc. Natl. Acad. Sci. USA 96, 4769 (1999).

[13] D. Kleckner and W. T. Irvine, Creation and dynamics of knotted vortices, Nat. Phys. 9, 253 (2013).

[14] H. Kedia, I. Bialynicki-Birula, D. Peralta-Salas, and W. T. M. Irvine, Tying Knots in Light Fields, Phys. Rev. Lett. 111, 150404 (2013).

[15] J. B. Taylor, Relaxation of Toroidal Plasma and Generation of Reverse Magnetic Fields, Phys. Rev. Lett. 33, 1139 (1974).

[16] R. L. Ricca and F. Maggioni, Groundstate energy spectra of knots and links: Magnetic versus bending energy, in New Directions in Geometric and Applied Knot Theory, edited by P. Reiter, S. Blatt, and A. Schikorra (De Gruyter, Berlin, Boston, 2018), pp. 276-288.

[17] L. Giomi, Ž. Kos, M. Ravnik, and A. Sengupta, Cross-talk between topological defects in different fields revealed by nematic microfluidics, Proc. Natl. Acad. Sci. USA 114, E5771 (2017).

[18] I. Muševič, M. Škarabot, U. Tkalec, M. Ravnik, and S. Žumer, Two-dimensional nematic colloidal crystals self-assembled by topological defects, Science 313, 954 (2006).

[19] U. Tkalec, M. Ravnik, S. Čopar, S. Žumer, and I. Muševič, Reconfigurable knots and links in chiral nematic colloids, Science 333, 62 (2011).

[20] T. Machon and G. P. Alexander, Global defect topology in nematic liquid crystals, Proc. R. Soc. A 472, 20160265 (2016).

[21] K. Sun, A. Souslov, X. Mao, and T. Lubensky, Surface phonons, elastic response, and conformal invariance in twisted kagome lattices, Proc. Natl. Acad. Sci. USA 109, 12369 (2012).

[22] D. Z. Rocklin, S. Zhou, K. Sun, and X. Mao, Transformable topological mechanical metamaterials, Nat. Commun. 8, 14201 (2017).

[23] Y. Kim, H. Yuk, R. Zhao, S. A. Chester, and X. Zhao, Printing ferromagnetic domains for untethered fast-transforming soft materials, Nature (London) 558, 274 (2018).

[24] J. Paulose, B. G.-g. Chen, and V. Vitelli, Topological modes bound to dislocations in mechanical metamaterials, Nat. Phys. 11, 153 (2015).

[25] A. Sengupta, C. Bahr, and S. Herminghaus, Topological microfluidics for flexible micro-cargo concepts, Soft Matter 9, 7251 (2013).

[26] R. Matthews, A. Louis, and J. Yeomans, Complex dynamics of knotted filaments in shear flow, EPL 92, 34003 (2010).

[27] B. Audoly, N. Clauvelin, and S. Neukirch, Elastic Knots, Phys. Rev. Lett. 99, 164301 (2007).

[28] M. K. Jawed, P. Dieleman, B. Audoly, and P. M. Reis, Untangling the Mechanics and Topology in the Frictional Response of Long Overhand Elastic Knots, Phys. Rev. Lett. 115, 118302 (2015).
[29] V. P. Patil, J. D. Sandt, M. Kolle, and J. Dunkel, Topological mechanics of knots and tangles, Science 367, 71 (2020).

[30] J. Cantarella, A. LaPointe, and E. J. Rawdon, Shapes of tight composite knots, J. Phys. A 45, 225202 (2012).

[31] V. Katritch, J. Bednar, D. Michoud, R. G. Scharein, J. Dubochet, and A. Stasiak, Geometry and physics of knots, Nature (London) 384, 142 (1996).

[32] H. K. Moffatt, The energy spectrum of knots and links, Nature (London) 347, 367 (1990).

[33] A. Y. Chui and H. K. Moffatt, The energy and helicity of knotted magnetic flux tubes, Proc. R. Soc. A 451, 609 (1995).

[34] B. Audoly, N. Clauvelin, P.-T. Brun, M. Bergou, E. Grinspun, and M. Wardetzky, A discrete geometric approach for simulating the dynamics of thin viscous threads, J. Comput. Phys. 253, 18 (2013).

[35] A. Goriely, Twisted elastic rings and the rediscoveries of Michell's instability, J. Elast. 84, 281 (2006).

[36] See Supplemental Material at http://link.aps.org/supplemental/ 10.1103/PhysRevResearch.2.043196 for a simulation movie.

[37] M. O'Neill and S. M. Kelly, Photoinduced surface alignment for liquid crystal displays, J. Phys. D 33, R67 (2000).

[38] J. Sun, A. K. Srivastava, L. Wang, V. G. Chigrinov, and H. S. Kwok, Optically tunable and rewritable diffraction grating with photoaligned liquid crystals, Opt. Lett. 38, 2342 (2013).

[39] Ž. Kos, M. Ravnik, and S. Žumer, Nematodynamics and structures in junctions of cylindrical micropores, Liq. Cryst. 44, 2161 (2017).

[40] A. N. Beris and B. J. Edwards, Thermodynamics of Flowing Systems with Internal Microstructure (Oxford University Press, New York, 1994).

[41] Y. Luo, D. A. Beller, G. Boniello, F. Serra, and K. J. Stebe, Tunable colloid trajectories in nematic liquid crystals near wavy walls, Nat. Commun. 9, 3841 (2018).

[42] A. Martinez, M. Ravnik, B. Lucero, R. Visvanathan, S. Žumer, and I. I. Smalyukh, Mutually tangled colloidal knots and induced defect loops in nematic fields, Nat. Mater. 13, 258 (2014).

[43] X. Wang, Y. Zhou, Y.-K. Kim, D. S. Miller, R. Zhang, J. A. Martinez-Gonzalez, E. Bukusoglu, B. Zhang, T. M. Brown, J. J. de Pablo, and N. L. Abbott, Patterned surface anchoring of nematic droplets at miscible liquid-liquid interfaces, Soft Matter 13, 5714 (2017).

[44] V. G. Chigrinov, V. M. Kozenkov, and H.-S. Kwok, Photoalignment of Liquid Crystalline Materials: Physics and Applications (Wiley, Chichester, 2008).

[45] G. P. Alexander, B. G. Chen, E. A. Matsumoto, and R. D. Kamien, Colloquium: Disclination loops, point defects, and all that in nematic liquid crystals, Rev. Mod. Phys. 84, 497 (2012).

[46] J. S. Lintuvuori, D. Marenduzzo, K. Stratford, and M. E. Cates, Colloids in liquid crystals: a lattice Boltzmann study, J. Mater. Chem. 20, 10547 (2010). 